09

\title{
Исследование температурной стабильности широкополосного электрооптического модулятора на основе микрорезонатора с модами шепчущей галереи
}

\author{
(C) К.Н. Миньков, ${ }^{1,2}$ А.С. Волошин, ${ }^{1}$ Н.М. Кондратьев, ${ }^{1}$ Е.А. Лоншаков, ${ }^{1,2}$ Р.В. Терентьев, ${ }^{1}$ В.Е. Лобанов, ${ }^{1}$ \\ Д.Ф. Зайцев, ${ }^{3}$ И.А. Биленко ${ }^{1}$ \\ ${ }^{1}$ Российский квантовый центр, \\ 121205 Москва, Россия \\ 2 Национальный исследовательский технологический университет „МИСиС“, \\ 119049 Москва, Россия \\ ${ }^{3} \mathrm{OAO}$ „Концерн „Вега“, \\ 121170 Москва, Россия \\ e-mail: k.n.minkov@yandex.ru
}

Поступило в Редакцию 19 ноября 2020 г.

В окончательной редакции 16 декабря 2020 г.

Принято к публикации 18 декабря 2020 г.

\begin{abstract}
Разработан электрооптический модулятор с полосой модуляции 0-900 MHz на основе микрорезонатора из ниобата лития с малой величиной полуволнового напряжения. Особое внимание уделено температурной стабильности созданного устройства. Предложены меры компенсации тепловых эффектов, позволившие уменьшить коэффициент теплового сдвига моды микрорезонатора. Получена зависимость сдвига частоты моды шепчущей галереи от мощности излучения, и найдено предельное значение допустимого изменения мощности накачки, составляющее для разработанного устройства $3.2 \mathrm{~mW}$.
\end{abstract}

Ключевые слова: микрорезонаторы, моды шепчущей галереи, СВЧ модуляторы.

DOI: $10.21883 /$ JTF.2021.06.50872.323-20

\section{Введение}

В современных системах связи, а также в радиолокации и радиоастрономии широко применяются различные устройства для детектирования, преобразования и обработки сигналов в СВЧ диапазоне (с частотами от единиц до десятков гигагерц). Радиофотонные устройства для обработки СВЧ сигналов в настоящее время получают все большее распространение ввиду того, что они обладают многими преимуществами по сравнению с системами на основе традиционной электроники. Во-первых, радиофотонные устройства обладают практически неограниченным быстродействием, а также высокой устойчивостью к внешнему электромагнитному излучению, что обусловлено фундаментальными различиями между фотонами и электронами как носителями энергии и информации. Во-вторых, переход работы аппаратуры с СВЧ диапазона в оптический диапазон позволяет существенно уменьшить массу и габариты разрабатываемых устройств. Подобный переход осуществляется при помощи электрооптических модуляторов [1], в которых происходит модуляция интенсивности оптического излучения СВЧ сигналом; поэтому электрооптические модуляторы (ЭОМ) являются одними из основных элементов различных радиофотонных систем. Кроме того, ЭОМ могут применяться для исследования различных квантовых эффектов [2].
В литературе было описано большое количество ЭОМ различных типов: на основе интерферометра Маха-Цендера [3-5], модуляторы на базе интегральных кольцевых резонаторов из кремния [6], а также резонаторов в форме микродиска [7], однако всем им свойственен недостаток, а именно большое значение полуволнового напряжения.

В настоящей работе представлена конструкция ЭОМ на основе микрорезонатора с модами шепчущей галереи (МШГ) [8-13]. Микрорезонаторы с модами шепчущей галереи представляют собой тело вращения из оптически прозрачного материала. Подобные резонаторы нашли широкое применение в различных областях науки и техники в качестве оптоэлектронных генераторов [14], датчиков для измерений свойств окружающей среды [15], датчиков магнитного поля [16] и сенсоров наночастиц [17]. Для изготовления микрорезонаторов пригодны материалы с высоким электрооптическим коэффициентом; наиболее часто применяются ниобат и танталат лития $\left(\mathrm{LiNbO}_{3}\right.$ и $\mathrm{LiTaO}_{3}$ соответственно). ЭОМ на основе микрорезонатора с МШГ может быть использован, в частности, в системах радиолокации, где необходимо обеспечивать прием слабых широкополосных сигналов мощностью $10^{-12}-10^{-13} \mathrm{~W}$ непосредственно от приемных антенн [7]. Обычно для этих целей между антенной и модулятором устанавливается малошумящий электронный усилитель (МШУ), что снижает динамический диапазон, увеличивает энергопотребление 
и делает всю систему незащищенной по отношению к различным электромагнитным помехам $[18,19]$. Необходимость установки МШУ, в свою очередь, объясняется высоким значением полуволнового напряжения (обычно несколько вольт) для большинства электрооптических модуляторов. Представленный в настоящей работе электрооптический модулятор на основе микрорезонатора с МШГ обладает низким полуволновым напряжением (менее $1 \mathrm{~V}$ ) и высокой чувствительностью в сочетании c широкой рабочей полосой частот модуляции, что дает возможность приблизиться к требованиям, предъявляемым к входным каскадам приемных устройств антенных решеток. Отметим, что в резонансных электрооптических модуляторах (типа МШГ) для общности с классическими модуляторами на эффекте Поккельса, полуволновое напряжение определяется по наклону зависимости сдвига фазы оптической волны от напряжения вблизи нуля (из-за нелинейности данной зависимости) при настройке на склон резонансной кривой. Эта величина оказывается зависимой от нагрузки МШГ модулятора и в перегруженном случае в $\pi / 4$ раз больше постоянного напряжения, при подаче которого происходит смещение резонансной частоты на ширину моды резонатора.

Преимущества использования радиофотонных устройств на основе микрорезонаторов с МШГ могут быть сведены на нет при отсутствии учета их температурной нестабильности [8]. Поэтому исследования температурной зависимости параметров устройств на основе микрорезонаторов с МШГ представляют большое практическое значение. Показано, что температурная нестабильность резонансной частоты в микрорезонаторах из диэлектрических материалов с отличным от нуля поглощением обусловлена в основном двумя причинами: изменением показателя преломления материала резонатора (терморефракция) и тепловым расширением этого материала при изменении температуры микрорезонатора. Изменение температуры микрорезонатора происходит, в частности, при поглощении части излучения, циркулирующего внутри резонатора. Для микрорезонатора из ниобата лития и коэффициент терморефракции, и коэффициент теплового расширения являются положительными величинами $\left(\{4.3 ; 4.3 ; 37.7\} \cdot 10^{-6} / \mathrm{K}\right.$ и $\{13.5 ; 13.5 ; 3.4\} \cdot 10^{-6} / \mathrm{K}$ соответственно [20]), что приводит к смещению резонансов в низкочастотную область при повышении температуры.

Целью настоящей работы являлось исследование температурной стабильности ЭОМ на основе микрорезонатора с МШГ, а также исследование основных характеристик ЭОМ. Для этого были исследованы зависимости резонансных частот МШГ от температуры конструкции модулятора и от мощности излучения накачки.

\section{1. Теоретические основы}

Преимуществом применения микрорезонаторов с модами шепчущей галереи в электрооптических моду- ляторах помимо высокой добротности является хорошее перекрывание взаимодействующих оптического и микроволнового полей. В работе [21] было проведено теоретическое моделирование работы ЭОМ на основе микрорезонатора из ниобата лития, в частности, было изучено электрооптическое взаимодействие между оптическими МШГ и СВЧ полем. Однако, поскольку пространственные размеры МШГ малы по сравнению с размерами, на которых электрическое поле СВЧ существенно меняется, то при расчете соответствующих интегралов перекрывания можно использовать его локальное значение в максимуме МШГ. Рассмотрим диэлектрический дисковый резонатор с металлическим напылением на верхнем и нижнем основании (здесь и далее вертикальным или аксиальным будем называть направление, перпендикулярное плоскости диска (и элемента связи), а горизонтальным или радиальным - в плоскости диска и элемента связи). Будем считать напыленное проводящее покрытие эквивалентным СВЧ резонатором. В этом случае для микрорезонатора из ниобата лития, считая вклад электрооптического коэффициента малым (в 10 раз меньше остальных), относительный частотный сдвиг МШГ равен

$$
\delta_{\omega} \approx \sum_{\lambda, n} \frac{e_{\lambda} r_{\lambda n}^{\mathrm{eff}} E_{n}}{2 \varepsilon_{s}^{-1}} \frac{\sin \left(\pi M l_{\varphi}\right)}{\pi M} \exp \left(i \pi M l_{\varphi}\right)
$$

где $E_{n}-$ компоненты вектора СВЧ поля в центре МШГ, $M=m_{p}-m_{s}+m_{\mathrm{RF}} / l_{\varphi}-$ комбинация азимутальных чисел накачки, сигнальной и СВЧ моды, $l_{\varphi}-$ относительная длина СВЧ-резонатора, $\varepsilon_{s}^{-1}$ - элемент обратной матрицы диэлектрической проницаемости сигнальной моды на оптической частоте, соответствующий ее поляризации (элемент „33“ для вертикальной поляризации и „11“ - для горизонтальной $), r_{\lambda n}^{\text {eff }}-$ компоненты электрооптического тензора ниобата лития с $r_{12}=0, e_{\lambda}=e_{i}^{s} e_{j}^{p}-$ шестимерный вектор произведения поляризаций оптических мод накачки и сигнала. Суммирование производится по всем компонентам СВЧ поля $(n=1-3)$ и компонентам поляризации $(\lambda=1-6)$. Считая, что поляризация МШГ строго вертикальная и что выполнены условия синхронизма $M=0$, получим простую формулу:

$$
\delta_{\omega} \approx \frac{1}{2} \varepsilon_{s} r_{33} E l_{\varphi} \cos \theta
$$

где $E$ и $\theta$ модуль вектора напряженности поля в центре МШГ и угол его направления с вертикалью, $\varepsilon_{s}$ - матричный элемент тензора диэлектрической проницаемости, соответствующий поляризации сигнальной оптической волны (элемент „33“ для вертикальной поляризации и „11“ - для горизонтальной). В случае ЭОМ на основе микрорезонатора из ниобата лития с проводящим напылением с двух сторон можно считать СВЧ поле полем конденсатора, включенного в схему 
согласования. Тогда его можно рассчитать по формуле

$$
E=\frac{1}{h} \frac{U_{0}}{1+i R C \Omega-\Omega^{2} L C},
$$

где $U_{0}-$ входное напряжение, $L-$ индуктивность схемы согласования, $R$ - ее сопротивление, $C=C_{0}(1-i \alpha)$ и $h-$ емкость с потерями и толщина микрорезонатора с напылением. Для микрорезонатора из ниобата лития толщиной $h=100 \mu \mathrm{m}$ и кольцевого электрода емкость равна порядка $1.2 \mathrm{pF}$, и тангенс угла потерь $\alpha=0.001$. На практике эффективность ЭОМ выражают полуволновым напряжением, в случае ЭОМ на основе микрорезонатора из ниобата лития с проводящим напылением с двух сторон от МШГ [21]

$$
V_{\pi}=\frac{\pi h E}{4 Q \delta_{\omega}}=\frac{\pi h}{2 Q \varepsilon_{s} r_{33} l_{\varphi} \cos \theta},
$$

где $Q$ - добротность микрорезонатора с МШГ. Для добротности $Q=10^{6}$, вертикальной поляризации оптического и СВЧ поля $\left(r_{33}=33 \mathrm{pm} / \mathrm{V}, \theta=0\right)$ и полнодискового электрода $l_{\varphi}=1$ получим оценку $V_{\pi}=0.5 \mathrm{~V}$.

Чтобы оценить амплитуду модуляции, используем методику расчета сигнала, представленную в [21]. Вначале колебание в каждой моде раскладывается по гармоникам частоты модуляции $E_{k}=\sum_{n} a_{k}^{(n)} e^{i n \Omega t} e^{i \omega t}$. Далее предполагается, что амплитуда некой гармоники под номером $\pm(N+1)$ мала и не требует учета. Тогда сначала рассчитывается рекуррентный набор определяющих матриц

$$
M_{n}^{\prime}=\Delta_{n}-n \Omega-i \frac{\kappa_{k j}}{2}-\mu_{-} \mu_{+} \delta_{\omega_{k j}}^{ \pm} M_{n \pm 1}^{\prime} \delta_{\omega_{k j}}^{\mp},
$$

начиная с $M_{ \pm N}^{\prime}=\Delta_{ \pm N} \mp M \Omega-i \frac{\kappa_{k j}}{2}$, где $\Delta_{k}=\omega_{k}-\omega-$ отстройка от резонанса МШГ, $\Omega$ и $\omega-$ частоты СВЧ модуляции и оптической несущей, $\kappa_{k j}-$ потери МШГ (диагональные члены связаны с добротностью $\kappa_{k k}=\frac{\omega_{k}}{Q_{k}}$, недиагональные - с межмодовым взаимодействием и близки к нулю), $\mu_{ \pm}=\frac{(\omega \mp \Omega)^{2}}{2 \omega}$ близко к единице, $\delta_{\omega_{k j}}^{+}=\delta_{\omega_{k j}}, \delta_{\omega_{k j}}^{-}=\delta_{\omega_{k j}}^{7}$, и верхний знак относится к $n>0$. Далее рассчитывается амплитуда, начиная с нулевой:

$$
\begin{gathered}
b_{k}=a_{k}^{(0)}=F B^{-1} \frac{T_{k}}{\tau_{k}}, \\
B=\Delta_{k}-\mu_{+} \mu_{-}\left(\delta_{\omega_{k m}}^{-} M_{-}^{i-1} \delta_{\omega_{n j}}^{+}+\delta_{\omega_{k m}}^{+} M_{+}^{i-1} \delta_{\omega_{n j}}^{-}\right)-i \frac{\kappa_{k j}}{2},
\end{gathered}
$$

где $T_{k}$ - амплитудный коэффициент пропускания света в МШГ, $\tau_{k}-$ время обращения света по резонатору, $F$ - амплитуда поля элемента связи в объеме резонатора. Далее амплитуды остальных гармоник

$$
a_{k}^{(n)}=M_{n}^{i-1} \mu_{ \pm} \delta_{\omega_{k i}}^{\mp} a_{j}^{(n \mp 1)} .
$$

Теперь пересчитаем полученные выражения по выходу $E_{\text {out }}=\left(1+\sum_{n} i T_{k} a_{k}^{(n)} e^{i n \Omega t}\right) e^{i \omega t}$, где $a_{k}^{(n)}-$ амплитуда моды $k$ на гармонике $n$. Тогда, используя квадратичный детектор и пренебрегая высшими гармониками, для сигнала на частоте модуляции получим

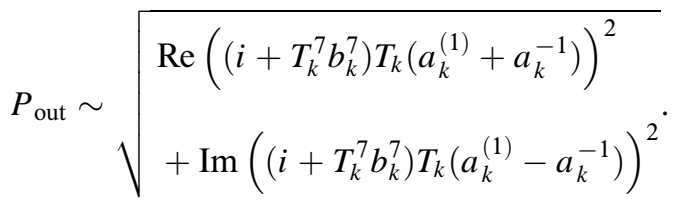

Заметим, что, так как коэффициент пропускания удобнее представить в виде $T_{k}=\sqrt{\eta_{k} \kappa_{k k} \tau_{k}}$, где $\eta_{k} \in[0 ; 1)-$ коэффициент связи [22], результат не будет зависеть от $\tau_{k}$. Обычно связь с резонатором настраивают таким образом, чтобы весь свет поглощался в резонаторе при резонансе, что соответствует $\eta_{k}=0.5$ (так называемая критическая связь). В электрооптических модуляторах также применяют перегруженные резонаторы, поскольку при этом уменьшается влияние тепловых эффектов (меньше мощность, поглощаемая резонатором) и расширяется диапазон рабочих частот, при этом, очевидно, полуволновое напряжение увеличивается.

При разработке ЭОМ на базе оптических микрорезонаторов следует учесть возможные нелинейные эффекты, связанные с высокой мощностью излучения накачки, а также с высокой мощностью СВЧ поля. При небольших мощностях входного излучения (менее $20 \mathrm{~mW}$ ) нелинейность отклика ЭОМ определяется зависимостью коэффициента модуляции от амплитуды сигнала. Для компенсации нелинейных эффектов в конструкции ЭОМ предусмотрена схема согласования.

\section{2. Конструкция широкополосного ЭОМ на основе микрорезонатора с МШГ и описание экспериментальной установки для исследования его характеристик}

Используя разработанную теорию и полученные ранее результаты численного моделирования [21,23], был разработан и создан прототип широкополосного ЭОМ с низким полуволновым напряжением. Фотография внутренних элементов ЭОМ показана на рис. $1, a$.

Излучение лазера накачки с длиной волны $1550 \mathrm{~nm}$ направлялось по входному оптическому волокну, сохраняющему плоскость поляризации лазерного излучения, в коллиматор 1. Конструктивно ЭОМ обеспечивает использование аксиально поляризованых мод, так как они имеют большую добротность. Далее излучение фокусировалось при помощи градиентной линзы 2 на призму 3 , изготовленную из рутила $\left(n_{\mathrm{TiO}_{2}}=2.865\right)$. Призма использовалась в качестве элемента связи с микрорезонатором с модами шепчущей галереи 4. Использовались микрорезонаторы дисковой формы, изготовленные из пластинок ниобата лития $\left(\mathrm{LiNbO}_{3}\right)$ толщиной $100 \mu \mathrm{m}$ с напылением проводящего слоя золота; добротность используемых микрорезонаторов составляла $10^{5}-10^{6}$ 


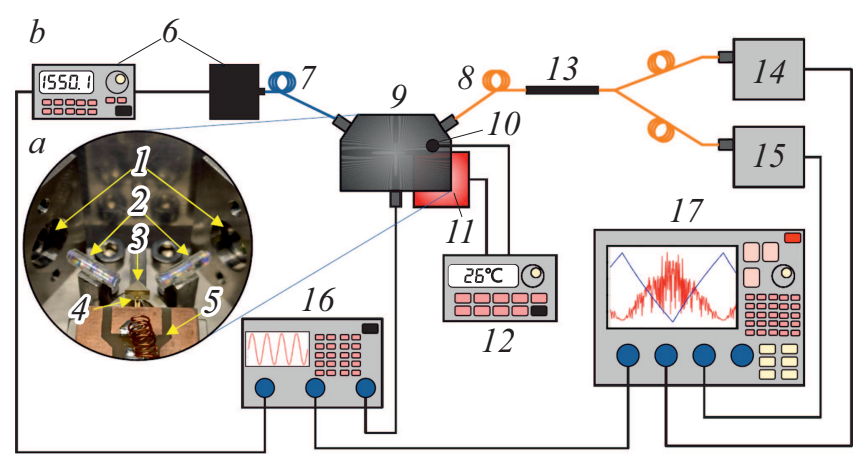

Рис. 1. $a-$ фотография ЭОМ на основе микрорезонатора с МШГ: 1 - коллиматоры, 2 - градиентные линзы, 3 - рутиловая призма, 4 - микрорезонатор с МШГ из ниобата лития, 5 - плата согласования, соединенная с СВЧ разъемом. $b$ - схема экспериментальной установки для исследования характеристик электрооптического модулятора на основе микрорезонатора с МШГ: 6 - блок управления лазером накачки $1550 \mathrm{~nm}, 7$ - оптическое волокно с сохранением поляризации, 8 - оптическое волокно, 9 - электрооптический модулятор, 10 - датчик температуры, 11 - элемент Пельтье, 12 - термоконтроллер, 13 - волоконный разветвитель $90: 10,14$ - медленный фотодетектор, 15 - быстрый фотодетектор, 16 - генератор СВЧ сигналов, 17 - осциллограф.

при величине области свободной дисперсии $45 \mathrm{GHz}$. Промодулированное излучение выводилось из микрорезонатора при помощи призмы, и направлялось на градиентную линзу, а затем на коллиматор, и далее по выходному оптическому волокну поступало на устройства для дальнейшей обработки сигнала. СВЧ сигнал модуляции от источника подавался через СВЧ разъем на плату согласования 5. На плате согласования была собрана схема, состоящая из резистора $200 \Omega$, подключенного параллельно СВЧ резонатору, и катушки, включенной последовательно с СВЧ резонатором. Индуктивность катушки $(10 \mathrm{nH})$ рассчитывалась таким образом, чтобы скомпенсировать нелинейность частотной характеристики модуляции на высших частотах рабочего диапазона 0-900 MHz.

В качестве СВЧ резонатора использовался металлический проводящий слой на поверхности микрорезонатора, в виде микрополоска шириной $300 \mu \mathrm{m}$. Проводящий слой на поверхности микрорезонатора был электрически соединен с платой согласования тремя серебряными проводниками толщиной $20 \mu \mathrm{m}$ при помощи ультразвуковой пайки.

Для исследования характеристик собранного модулятора нами была собрана экспериментальная установка, схема которой изображена на рис. $1, b$. Излучение лазера накачки Koheras Adjustik 6 с центральной частотой $193.5 \mathrm{THz}$, перестраиваемой в диапазоне $1 \mathrm{GHz}$, и шириной полосы излучения $10 \mathrm{kHz}$, а также с перестраиваемой мощностью выходного излучения, направлялось на вход электрооптического модулятора 10 через опти- ческое волокно PM-S350-HP, Thorlabs 7, сохраняющее плоскость поляризации.

При измерении амплитудно-частотной характеристики модулирующий СВЧ сигнал подавался на электрооптический модулятор с генератора KeySight N5173B 16, с рабочей полосой частот от $9 \mathrm{kHz}$ до $1 \mathrm{GHz}$. Выходящее из модулятора промодулированное излучение разделялось с помощью волоконного разветвителя 90:10 TN1550R2A1, Thorlabs 13 на два пучка, более мощный из которых направлялся на быстрый фотодетектор FPD310, Menlo Systems 15 с полосой пропускания 10-1000 MHz, а менее мощный - на медленный фотодетектор PDA50B-EC, Thorlabs 14 с полосой пропускания 0-460 kHz. Оба фотодетектора были подключены к осциллографу KeySight InfiniiVision MSO-X 4154A 17. При помощи цифрового осциллографа с полосой пропускания $1.5 \mathrm{GHz}$ осуществлялось измерение параметров входного и выходного сигналов электрооптического модулятора, а также сбор данных.

\section{3. Характеристики ЭОМ}

Нами были исследованы основные характеристики созданного ЭОМ. В первую очередь была изучена амплитудно-частотная характеристика (АЧХ) ЭОМ в диапазоне частот $10-1000 \mathrm{MHz}$ (рис. 2). Для измерения АХЧ модулятора в качестве источника излучения использовался перестраиваемый лазер VitaWave QDFBLD-1550-50 с шириной полосы излучения $100 \mathrm{kHz}$. Для измерения амплитуды модуляции лазерного излучения использовался быстрый фотодетектор Menlo Systems FPD 310. В AЧX заметен резонанс схемы

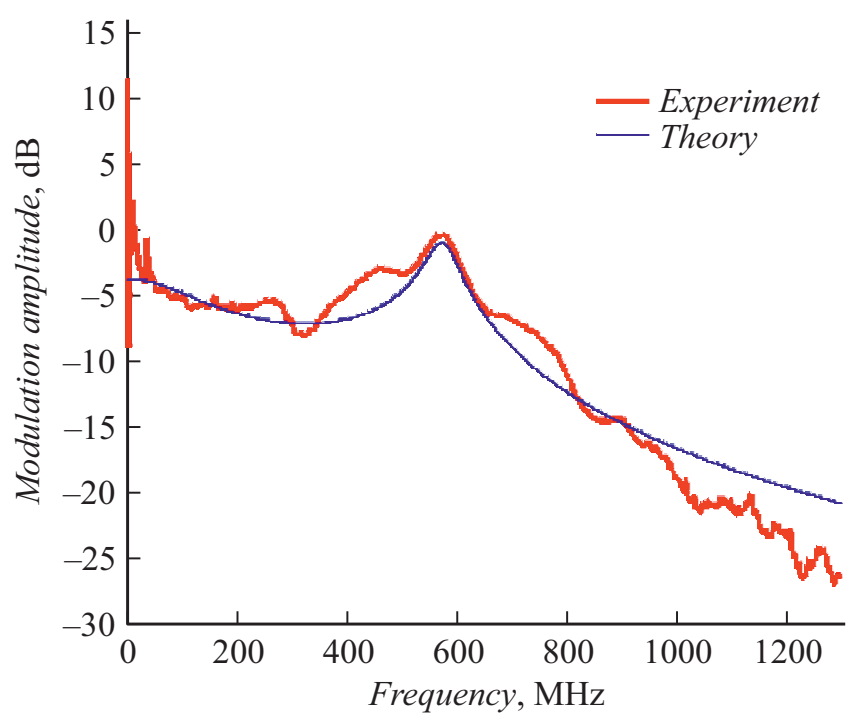

Рис. 2. АХЧ электрооптического модулятора из инвара (36Н). Красная кривая (в online версии) - эксперимент, синяя линия (в online версии) - предсказание теории (2), (3), (5)-(9) при $L=64 \mathrm{nH}$ сопротивлением $R=10 \Omega$, оптической добротности $Q=10^{6}$. Синяя кривая перенормирована на высоту СВЧ резонанса. 
СВЧ согласования. Факт наличия данного резонанса также был проверен при помощи измерителя КСВ. Применение резонансной схемы согласования позволило расширить диапазон рабочих частот модуляции в области высоких частот до $900 \mathrm{MHz}$ по уровню $-15 \mathrm{~dB}$ (рис. 2). Отметим, что полученная АЧХ хорошо описывается разработанной теоретической моделью. Следует отметить, что расширение диапазона уменьшает значение коэффициента стоячей волны; в созданном нами электрооптическом модуляторе с описанной выше схемой согласования значение коэффициента стоячей волны составляло не более 2 во всем рабочем диапазоне частот модуляции.

Также были определены характеристики, играющие наиболее важную роль при его эксплуатации, в частности, было измерено эффективное полуволновое напряжение на краях рабочего диапазона - не более $0.5 \mathrm{~V}$, а также определена величина полных оптических потерь в электрооптическом модуляторе, которая составила не более $4 \mathrm{~dB}$. Также в ходе экспериментов нами не было обнаружено проявлений нелинейности отклика ЭОМ при входных сигналах амплитудой до $10 \mathrm{~V}$, что объясняется выбором рабочей точки на линейном участке передаточной функции [21].

\section{4. Термостабилизация ЭОМ}

Для микрорезонатора ЭОМ сдвиг резонансной частоты МШГ $\Delta \omega$, вызванный изменением температуры $\Delta T[24]$, может быть оценен как

$$
\Delta \omega=-\omega_{0}\left(\alpha_{T}+\frac{1}{n} \frac{d n}{d T}\right) \Delta T,
$$

где $\alpha_{\tau}-$ коэффициент теплового расширения (в радиальном направлении), $n$ - показатель преломления материала резонатора. Величина $\frac{d n}{d \tau}$ в выражении (10) называется коэффициентом терморефракции и, как и показатель преломления, зависит от поляризации света. Для микрорезонатора из ниобата лития, использующегося в электрооптическом модуляторе, представленном в настоящей работе, теоретическая оценка ухода частоты, рассчитанная по формуле $(10)$, составляет $3 \mathrm{GHz} / \mathrm{K}$ для радиальных мод (определяясь в основном тепловым расширением), и $6 \mathrm{GHz} / \mathrm{K}$ для аксиальных мод (определяясь в основном терморефракцией).

Контроль температурного режима ЭОМ осуществлялся при помощи LDC500, Stanford Research Systems (элемент 12 на рис. 1), который подключался к датчику температуры AD592 (элемент 10 на рис. 1), закрепленному на плите внутри корпуса модулятора. Изменение температуры производилось при помощи элемента Пельтье (элемент 11 на рис. $1, b)$, установленного между модулятором и оптическим столом.

Была получена зависимость резонансной частоты выбранной моды микрорезонатора от температуры. Для этого были изучены спектры МШГ микрорезонатора в
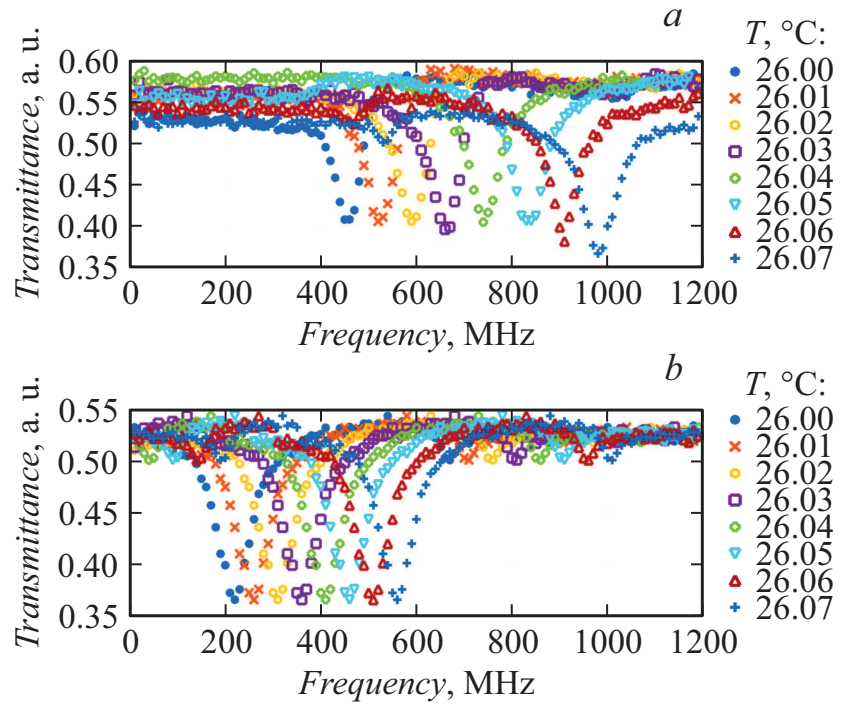

Pис. 3. $a-$ спектры МШГ модулятора, изготовленного из дюралюминия (Д16Т), полученные при изменении температуры модулятора на $0.1^{\circ} \mathrm{C} . b-$ спектры МШГ модулятора, изготовленного из инвара $(36 \mathrm{H})$, полученные при изменении температуры модулятора на $0.1^{\circ} \mathrm{C}$. Цветом показаны спектры при различных температурах.

процессе нагревания ЭОМ. Сначала температура модулятора стабилизировалась с помощью термоконтроллера в начальной точке. После этого на термоконтроллере устанавливалась температура на $0.1^{\circ} \mathrm{C}$ выше начальной; спектры МШГ регистрировались каждые $30 \mathrm{~s}$ в течение $10 \mathrm{~min}$, начиная с момента запуска изменения температуры на термоконтроллере. При изменении температуры более чем на $0.2^{\circ} \mathrm{C}$ выбранная мода смещалась за пределы диапазона плавной перестройки частоты используемого лазера Koheras Adjustik (1000 MHz). Спектры МШГ модулятора, основание и конструктивные элементы которого были изготовлены из дюралюминия (Д16Т), полученные в процессе изменения температуры модулятора на $0.1^{\circ} \mathrm{C}$, представлены на рис. $3, a$.

Исходя из значений сдвига резонансных частот мод в зависимости от температуры в начале и в конце температурной перестройки на \pm 0.1 и $\pm 0.2^{\circ} \mathrm{C}$ (относительно начальной температуры $26.00^{\circ} \mathrm{C}$ ) был определен коэффициент данной зависимости, величина которого оказалась равной $7.5 \mathrm{GHz} / \mathrm{K}$, немного больше оценки по формуле (10) для аксиальных мод резонатора $(6 \mathrm{GHz} / \mathrm{K})$. Также на рис. 3, a можно увидеть явное изменение амплитуды моды, что может быть связано с нарушением юстировки ЭОМ.

Сдвиг частоты, вызываемый флуктуациями температуры МШГ, не должен превышать полуширину моды (около $100 \mathrm{MHz}$ для добротности $10^{6}$ ). При получении спектров частота лазера сканировалась в диапазоне 0-1000 MHz относительно частоты $193.524 \mathrm{THz}$. Коэффициент линейной зависимости на рис. $4, b$ составляет $33 \mathrm{MHz} / \mathrm{mW}$. Принимая во внимание величину коэф- 
фициента температурной зависимости частоты МШГ, можно оценить величину локального нагрева материала микрорезонатора, обусловленного поглощением излучения: $6.2 \cdot 10^{3} \mathrm{~K} / \mathrm{mW}$. Пользуясь значением предельно допустимого отклонения температуры микрорезонатора, полученным выше для инварового ЭОМ $\left( \pm 0.02^{\circ} \mathrm{C}\right)$, можно оценить предельно допустимое изменение мощности лазерного излучения накачки $- \pm 3.2 \mathrm{~mW}$. Таким образом, представленный ЭОМ имеет сильную температурную зависимость частоты мод.

Для повышения термостабильности устройств были предприняты следующие меры: все детали конструкции, изготовленные ранее из дюралюминия (Д16Т), были заменены на детали из инвара $(36 \mathrm{H})$ - материала, обладающего низким $\left(1.2 \cdot 10^{-6} / \mathrm{K}\right)$ температурным коэффициентом расширения при нормальных условиях (ср. $22.9 \cdot 10^{-6} / \mathrm{K}$ для Д16Т). Было выдвинуто предположение, что при нагреве возникают малые деформации конструкции модулятора. Эти деформации, в свою очередь, приводят к изменению согласования входного светового пучка с модой резонатора. Как следствие, изменяется мощность света внутри резонатора и его резонансные частоты. Применение материала с малым коэффициентом теплового расширения должно существенно снизить вклад этого эффекта. Для проверки этой гипотезы было проведено измерение температурного коэффициента для модулятора с деталями из дюралюминия и инвара. Эксперимент показал, что при применении инвара температурный коэффициент снизился до $5.3 \mathrm{GHz} / \mathrm{K}$ и уменьшилось изменение коэффициента пропускания модулятора с температурой (ср. рис. $3, a$ и $b$ ).

Также была изучена зависимость спектра мод микрорезонатора от мощности входного лазерного излучения накачки. Поскольку температура микрорезонатора меняется при изменении мощности излучения накачки, следует ожидать, что изменение мощности вызывает сдвиг частот мод шепчущей галереи. Спектры МШГ и полученная зависимость частоты моды от мощности излучения накачки для ЭОМ из инвара $(36 \mathrm{H})$ представлены на рис. 4.

Существуют методы, которые могут позволить дополнительно повысить термостабильность ЭОМ. Значительное улучшение может быть достигнуто применением термокомпенсированного резонатора. Методы достижения этого сводятся в основном к помещению микрорезонатора в среду, обладающую иными, чем материал микрорезонатора, коэффициентами теплового расширения и терморефракции. Наиболее часто применяются тонкие пленки из полимерных материалов, обладающих отрицательным коэффициентом терморефракции $[25,26]$. В работе [27] теоретически и экспериментально исследовалась компенсация температурных сдвигов резонанса в зависимости от радиуса резонатора при изменении коэффициента терморефракции среды, окружающей резонатор. В качестве среды использовались водные растворы глицерина различной концентрации. Данный метод отличается простотой и не требует высокой точности
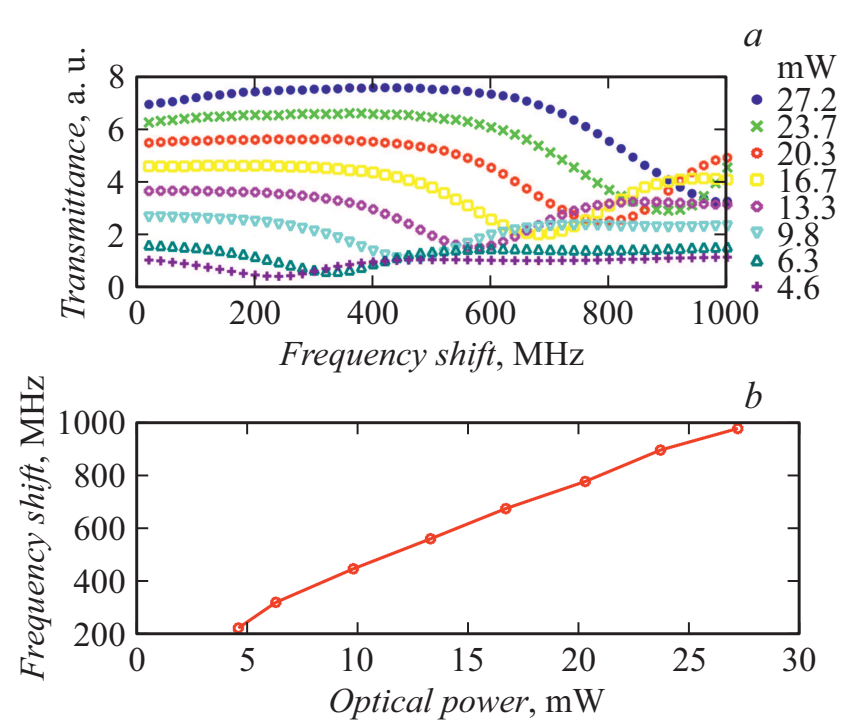

Рис. 4. $a-$ спектры МШГ, полученные при различной мощности излучения лазера накачки; $b-$ зависимость сдвига частоты МШГ от мощности излучения накачки.

изготовления, как того требуют методы, основанные на нанесении тонких полимерных пленок [24,26]. Также термокомпенсация достигается за счет использования специально разработанной конструкции композитного резонатора. Для микрорезонаторов из фторида магния была разработана сэндвичевая структура со слоями из церодура (Zerodur), что привело к уменьшению чувствительности к флуктуациям в 7 раз [28]. Для фторида кальция применение композитной структуры со слоями церодура обеспечивало уменьшение чувствительности к флуктуациям в 3 раза. Однако для фторида кальция более перспективным оказалось создание композитной структуры со слоями из керамики с отрицательным коэффициентом теплового расширения [29]. Такой подход позволил уменьшить чувствительность к флуктуациям более чем в 100 раз. Разработка оптимальной структуры термоустойчивых резонаторов из ниобата лития требует дальнейших исследований. Разрабатываются также и активные методы термостабилизации. В частности, с помощью кросс-поляризованной двухмодовой температурной стабилизации для двулучепреломляющего высокодобротного резонатора МШГ достигнут уровень неустойчивости температуры резонатора в $10 \mu \mathrm{K}$ даже до времени интегрирования $1000 \mathrm{~s}$ [30].

\section{Заключение}

Нами был создан ЭОМ на основе микрорезонатора с МШГ, отличающийся широкой полосой рабочих частот модуляции - до $900 \mathrm{MHz}$. Была создана экспериментальная установка для исследования различных характеристик созданного модулятора, а также для получения спектров МШГ. Была получена АХЧ модулятора. Величина полных оптических потерь в электро- 
оптическом модуляторе не превышает $4 \mathrm{~dB}$. Отметим, что предложенная нами система вариативна и имеет несколько путей конструктивного улучшения в зависимости от конкретной задачи. Так, можно отметить, что уменьшение толщины резонатора позволит уменьшить значение полуволнового напряжения, однако приведет к увеличению температурной чувствительности, которая в свою очередь может быть скомпенсирована за счет использования лазера накачки с быстрой перестройкой частоты (при значениях $10 \mathrm{~Hz} / \mathrm{s}$ и выше система сможет компенсировать практически любой дрейф внешней температуры), и системы релокинга рабочей точки, для которой могут быть предложены различные методы контроля состояния модулятора (такие как контроль температуры резонатора или резонансной частоты), которые также могут быть оптимизированы исходя из конкретной задачи.

Было изучено влияние температуры конструкции модулятора и мощности излучения накачки на резонансные частоты МШГ микрорезонатора из ниобата лития. Показано, что микрорезонатор из ниобата лития имеет сильную зависимость резонансных частот МШГ от температуры. Также показано, что температурные характеристики ЭОМ зависят не только от материала и структуры резонатора, но и от всей конструкции в целом. В частности, замена деталей из дюралюминия на детали из инвара позволило существенно увеличить термоустойчивость прибора. Также был определен коэффициент зависимости резонансной частоты МШГ от оптической мощности излучения накачки $33 \mathrm{MHz} / \mathrm{mW}$ и рекомендуемая стабильность этой мощности $\pm 3.2 \mathrm{~mW}$. Предложены перспективные активные и пассивные методы меры компенсации тепловых эффектов ЭОМ.

\section{Благодарности}

Работа выполнена с использованием оборудования ЦКП ВНИИОФИ (ckp.vniiofi.ru).

\section{Финансирование работы}

Работа поддержана Российским научным фондом (проект № 20-12-00344).

\section{Конфликт интересов}

Авторы заявляют, что у них нет конфликта интересов.

\section{Список литературы}

[1] K. Liu, C.R. Ye, S. Khan, V.J. Sorger. Laser Photon. Rev., 9 (2), 172 (2015). https://doi.org/10.1002/lpor.201400219

[2] M. Tsang. Phys. Rev. A - At. Mol. Opt. Phys., 81 (6), 1 (2010). https://doi.org/10.1103/PhysRevA.84.043845

[3] A. Liu, R. Jones, L. Liao, D. Samara-Rubio, D. Rubin, O. Cohen, R. Nicolaescu, M. Paniccia. Nature, 427 (6975), 615 (2004). DOI: $10.1038 /$ nature02310
[4] L. Liao, D. Samara-Rubio, M. Morse, A. Liu, D. Hodge, D. Rubin, U.D. Keil, T. Franck. Opt. Express, 13 (8), 3129 (2005). https://doi.org/10.1364/OPEX.13.003129

[5] G.T. Reed, G. Mashanovich, F.Y. Gardes, D.J. Thomson. Nat. Photonics, 4 (8), 518 (2010). https://doi.org/10.1038/nphoton.2010.179

[6] Q. Xu, B. Schmidt, S. Pradhan, M. Lipson. Nature, 435 (7040), 325 (2005). DOI: 10.1038/nature03569

[7] T. Sadagopan, S.J. Choi, S.J. Choi, P.D. Dapkus, A.E. Bond. IEEE Photon. Technol. Lett., 17 (3), 567 (2005). DOI: $10.1109 /$ LPT.2004.842365

[8] T. Sadagopan, Seung June Choi, Sang Jun Choi, P.D. Dapkus, A. Bond. „High-speed, low-voltage modulation in circular WGM microresonators," in Digest of the LEOS Summer Topical Meetings Biophotonics/Optical Interconnects and VLSI Photonics/WBM Microcavities, (2004), c. 7. DOI: 10.1109/LEOSST.2004.1338728

[9] A.A. Savchenkov, W. Liang, A.B. Matsko, V.S. Ilchenko, D. Seidel, L. Maleki. „Tunable resonant single-sideband electro-optical modulator," in 2009 IEEE/LEOS Summer Topical Meeting, 1, 63 (2009). https://doi.org/10.1364/OL.34.001300

[10] L. Maleki, A.F.J. Levi, S. Yao, V. Ilchenko, US006473218B1, (2002).

[11] D.A. Cohen, A.F.J. Levi. Solid. State. Electron., 45 (3), 495 (2001). https://doi.org/10.1016/S0038-1101(00)00248-3

[12] L. Maleki, A.B. Matsko, A. Savchenkov, V. Ilchenko, D. Seidel. Tunable single sideband modulators based on electro-optic whispering gallery mode resonators and their applications, US 2010/0118375 A1, (2010).

[13] A.B. Matsko, A. Savchenkov, D. Seidel, L. Maleki, V. Ilchenko, US 8331008 B1, (2012).

[14] V.B. Braginsky, M.L. Gorodetsky, V.S. Ilchenko. Phys. Lett. A, 137 (7-8), 393 (1989). https://doi.org/10.1016/0375-9601(89)90912-2

[15] M.L. Gorodetskii, Y.A. Demchenko, D.F. Zaitsev, V.N. Krutikov, Y.M. Zolotarevskii, V.L. Lyaskovskii. Meas. Tech., 57 (12), 1386 (2015). https://doi.org/10.1007/s11018-015-0639-9

[16] A. Mahmood, V. Kavungal, S.S. Ahmed, G. Farrell, Y. Semenova, Opt. Lett., 40 (21), 4983 (2015). https://doi.org/10.1364/OL.40.004983

[17] A.D. Ivanov, K.N. Min'kov, A.A. Samoilenko, D.D. Ruzhitskaya. Measurement Techniq., 6, (2018). DOI: $10.1007 / \mathrm{s} 11018-018-1466-6$

[18] M. Hossein-Zadeh, A.F.J. Levi. IET Optoelectron., 5 (1), 36 (2011). DOI: 10.1049/iet-opt.2009.0100

[19] V. Ilchenko, M. Gorodetsky. Laser Phys., 2, (1992). https://www.researchgate.net/publication/248076229_Thermal _nonlinear_effects_in_optical_whispering_gallery microresonators

[20] R.S. Weis, T.K. Gaylord. Appl. Phys. A Solid. Surf., 37 (4), 191 (1985). https://doi.org/10.1007/BF00614817

[21] N.G. Pavlov, N.M. Kondratyev, M.L. Gorodetsky. Appl. Opt., 54 (35), 10460, (2015). https://doi.org/10.1364/AO.54.010460

[22] M.L. Gorodetsky, V.S. Ilchenko. J. Opt. Soc. Am. B, 16, 147 (1999). https://doi.org/10.1364/JOSAB.16.000147

[23] N.M. Kondratiev, M.L. Gorodetsky. Bull. Russ. Acad. Sci. Phys., 77 (12), 1432 (2013). https://doi.org/10.3103/S1062873813130078

[24] I. Grudinin, H. Lee, T. Chen, K. Vahala. Opt. Express, 19 (8), 7365 (2011). https://doi.org/10.1364/OE.19.007365 
[25] C. Xie, D. Cui, J. Tang, C. Shang, T. Zhang, C. Xue, J. Liu., Micro Nano Lett., 9 (9), 609 (2014). https://doi.org/10.1049/mnl.2014.0223

[26] L. He, Y.F. Xiao, C. Dong, J. Zhu, V. Gaddam, L. Yang. Appl. Phys. Lett., 93 (20), 1 (2008). https://doi.org/10.1063/1.3030986

[27] E. Kim, M.R. Foreman, M.D. Baaske, F. Vollmer. Appl. Phys. Lett., 106 (16), (2015). https://doi.org/10.1063/1.4918932

[28] J. Lim, A.A. Savchenkov, E. Dale, W. Liang, D. Eliyahu, V. Ilchenko, A.B. Matsko, L. Maleki, C. Wei Wong. Nat. Commun., 8, 8 (2017). https://doi.org/10.1038/s41467-017-00021-9

[29] A. Savchenkov, A. Matsko. J. Opt., 20, 035801 (2018). https://orcid.org/0000-0001-9939-9311

[30] J. Lim, Wei Liang, A.A. Savchenkov, A.B. Matsko, L. Maleki \& C. Wei Wong., Light. Science \& Application, 8, 1 (2019), https://doi.org/10.1038/s41377-018-0109-7 